\title{
Tailoring anatase nanotubes for the photovoltaic device by the anodization process on behalf of
} microstructural features of titanium thin film

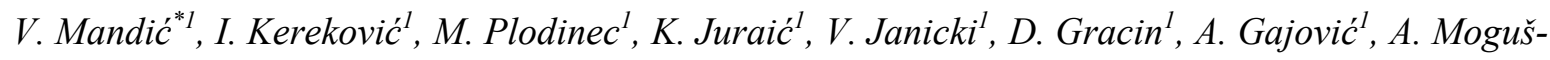 \\ Milankovic ${ }^{1}$, M.G. Willinger ${ }^{2}$ \\ ${ }^{1}$ Ruđer Bošković Institute, 54 Bijenička cesta, HR-10 000 Zagreb, Croatia \\ ${ }^{2}$ Fritz Haber Institute of the Max Planck Society, Faradayweg 4-6, D-14195 Berlin, Germany
}

\begin{abstract}
We prepared the anatase nanotubes (NT) and other nanostructured titania as electron accepting/transmitting layers in solar cells, using titanium anodization. Upon gaining control over the anodization, the parameters were held constant in order to observe the role of the deposited layers (by electron beam evaporation and magnetron sputtering) on the NT yield. The structural and microstructural parameters were investigated using FIB-FEG-SEM, XRD, Raman and GIXRD. Differences in the titanium layers play a significant role on the type of titania nanostructures achieved. Only dense homogeneous titanium layer surface, uniform in thickness and without cracks at macroscale, with uniformly seized isotropic nanoparticles, will enable uniform electrochemical etching and thus favourable, reproducible formation of the titania NT, and upon thermal treatment the anatase NT. Transparent conductive oxide (TCO) layers, which are not in direct contact to the NT, can also exhibit influence on the morphology of the titania NT charge transfer layer. In order to reach large uniform areas of nanotubes, which is required for solar cell application, the preparation of titanium layer has to be tailored.
\end{abstract}

\section{Keywords:}

Titanium; Electron beam evaporation; Magnetron sputtering; Anatase nanotubes; Anodization; Charge transfer solar cell material.

*Corresponding author: Vilko Mandić, e-mail:vmandic@irb.hr,vmandic@fkit.hr,tel: +3851 4571272 


\section{Introduction}

Photovoltaic devices seem to be the most lucrative answer (clean, free, unlimited, low maintenance, etc.) to the large demands of the future [1]. In a $3^{\text {rd }}$ generation photovoltaic device, like dye sensitized solar cell (DSSC), or perovskite based solar cell (PSC), electron transmitting material (ETM) layer is considered to be the curtail for enabling high efficiency in the overall performance of solar cell $[2,3]$. Namely, when excited by the light active material will yield excitons and the electron transmitting material which is underlaying the active material will ensure separation of the charge carriers and their further transfer to the electrode in order to finally yield electricity. The better the process, the higher performance of overall solar cell is achieved. The problem lies within the short lifetime of the photogenerated charge carriers, therefore the layer has to remain thin. Implementation of various nanostructural forms of the buffer layers ensures the increase of specific surface area, and enables more efficient charge transfer without the need to increase the thickness of the layers. Majority of non-inverted solar cells (SC) repose on the transparent conductive oxide (TCO) layer in order to serve as a transparent electrode underlying the electron transmitting material. Actually, the interface between the overlaying charge transfer oxide (CTO) layer and the underlaying transparent conductive oxide (TCO) layer is concerned as one of the critical parameters in active material-to-electrode electron transport. The main disadvantageous process in the solar cells is the non-radiative recombination which is more probable at the interfaces of grains in the active material, but will also occur at the defect states in bulk of the active materials. Having in mind a multi-layer assembly of a solar cell, limits in the control of the interfaces can be nominated as one of the main reasons why nowadays all (familiar) types of solar cell (SC) materials still fail to get closer to the theoretical efficiency limits.

While literature agrees that the most important task is the optical, electrical and structural compatibility of active and passive layers, there is no consensus on mechanisms of processes at boundaries (for example charge transfer, charge recombination, etc.) which cause discrepancies in optimisation efforts [1-22]. Thus, in the bottom-top solar cell assembly processes, much attention is devoted to the strategy of overlaying the CTO layers. The upgrade in efficiency of modern solar cell is 
known to depend not only on the chemical composition of the active material but also significantly on the morphology of materials at the interface between active and transport layers. Having in mind the chemical composition, titania is recognised as photoactive, photocatalytic, semiconducting material with known application as both charge transfer and active absorbing material [4]. In the case of special morphology, i.e. tailored self-organisation, for the titania thin films one can expect very favourable charge transfer properties, for example in the case of titania nanotube based thin films [5]. Such thin film can show significant enhancement of solar cell properties when used as an electron transmitting material, i.e. when placed between the absorbing material and the conductive transparent oxide layer, i.e. electrode. When compared to a nanoparticle film, $\mathrm{TiO}_{2}$ nanotubes (NT) have a large area-tovolume ratio, superior lifetime and provide optimal pathways for electron percolation, thus the ability to produce ordered self-assembled anatase nanotubes presented a huge milestone [6]. A $25 \%$ increase in the charge collection efficiency of nanotube-based solar cell when compared to nanoparticle-based solar cells occurs due to the enhanced light scattering and slower electron recombination [7]. Namely, morphology and self-organisation of CTO ensure enlarged specific surface area, i.e. contact to active material, while CTO remains thin enough to overcome the problem of the short lifetime of photogenerated charge carriers. The use of various nanostructural forms of inorganic materials in different crystal sizes in hybrid solar cells may allow additional tuning of the optical and electrical properties. The literature nominates various nano structures like tubes, rods, wires, fibers, particles, etc. [8]. Furthermore, tailoring of the optical gap and surface chemistry is employed to enhance the transfer of photo-generated carriers towards the external circuit. Currently, efficiencies of up to $13 \%$ were achieved for the titania NT-based DSSC, while above $20 \%$ for the NT-based PSC $[9,10]$. It is important to point out that these materials are the most cost effective solar cell constituent layers, both in terms of material and preparation (both laboratory and scale up) costs vs. power conversion efficiency. Subsequently, all of the above-mentioned influence the efficiency/stability of SC.

Layer deposition techniques also play an important role in the previously mentioned high efficiency separation of charge carriers and their transport from the absorbing material to the electrode. Achieving affordable and reliable deposition technique is the continuous matter of scientific effort [11- 
15]. Preparing the titania nanotube layer reposes on the starting Ti-layer. The successfulness of $\mathrm{Ti}$ deposition (with respect to structure, morphology, thickness, particle size, uniformity, etc.), anodization (with respect to deposition parameters), and thermal treatment (with respect to anodization parameters) is a necessity for the homogeneous surface which is needed for the solar cell application. In this case, a fully controllable and reproducible preparation procedure on all type of substrates still presents a challenge [16-18]. Namely, the Ti anodization process was introduced as a convenient way to produce the $\mathrm{TiO}_{2} \mathrm{NT}$ with various geometries in terms of nanotube length, diameter and wall thickness (basically a electrochemical etching process of the titanium in the ammonium fluoride electrolyte solution of various concentrations under different currents, where intermediate titanium fluoride is dissoluted to form amorphous titania nanotubes) [19-29], whereas the subsequent thermal treatment is required to promote the crystal transformation to anatase [30]. The process depends on many parameters, such as the $\mathrm{F}^{-}$ion concentration [31,32], the type of solvent [33], temperature [34,35], stirring [36] and anodization potential [34,37]. Yet anodization voltage and ammonium fluorite solution concentration are nominated as the most important. By changing these parameters, the nanotube parameters can be controlled, i.e. inner and outer tube diameters and the wall thickness [25]. The easiest way to control the length of the tubes is through the duration of the anodization process $[38,39]$. Very low water content in polar organic solvents may considerably decrease the rate of chemical dissolution and thus increase the nanotube length $[40,41]$. The presented anodization process was generalised as simple and facile. Namely, it was usually performed on commercial titanium foils. Such titanium materials were usually homogeneous with well-defined morphologies. XRD of such material yields the titanium with hexagonal crystal lattice. The need to produce the anatase nanotubes on different substrates, like the transparent substrates, brings about the problems in their preparation in micro- and macroscopically uniform manner [42]. In the literature, much attention is dedicated to the geometry of anodization derived $\mathrm{TiO}_{2} \mathrm{NT}$ layers, as it is presumed it is mainly responsible for the solar cell performance efficiency. The use of anatase nanotubes in solar cells is known to be more favourable in the non-inverted setup, meaning the light enters the cell on the titania side. Common substrate that ensures mechanical strength for the cell and transparency is glass. On top of glass one should have transparent electrode. For a transparent conductive oxide layer, aluminium doped zincite 
(AZO) is commonly used. Alloy of indium tin oxide (ITO) and fluorine doped tin oxide (FTO) are renowned candidates as well. In order to produce the nanotubes, it is necessary to deposit a thin film of titanium on top of TCO layer. Understanding of the transport mechanisms in titania nanotubes (with respect to their geometry), as well as the reproducibility of the preparation process (on all type of substrates) are still a matter of controversy and act as a milestone in the future upgrade of the titania NT-based solar cell performance $[39,18]$.

In this work, the influence of titanium layer (structure, morphology, uniformity, thickness, etc.) deposited on the TCO layer, on formation of well-defined homogeneous, ordered, self-organised, selfstanding titania nanotubes was studied in terms of the morphology of achieved nanotubes and reproducibility of the anodization process. The use of various nanostructured arrays may allow additional tuning to ensure the overall compatibility of all layers. The controlled deposition of the starting titanium layer was shown to be a prerequisite for successful anodization. The results of this work show that interface properties of layers underlaying the derived titania nanotubes (TCO, Ti) may significantly attribute to the behaviour of the solar cell.

\section{Experimental}

\subsection{Synthesis parameters}

The syntheses of porous $\mathrm{TiO}_{2}$ structures or $\mathrm{TiO}_{2}$ nanotubes by the anodization process were performed on Ti layer deposited by electron beam evaporation or magnetron sputtering under marginally different conditions on glass substrate with TCO layer. Thereafter, the prepared titanium samples were microstructurally and structurally characterised. Subsequently, the titanium thin films were anodized to titania nanotubes and thermally treated to anatase nanotubes (all under the same conditions) (Figure 1) and then microstructurally and structurally characterised again. The only process parameters that were differed are brought up in Table 1. Few steps were performed during the preparation: 
Glass substrates with transparent conductive electrode (aluminium zincite - AZO, Aldrich, United Kingdom; indium tin oxide - ITO, Ossila, United Kingdom; fluorine tin oxide - FTO, Aldrich, United Kingdom) for the depositions of titanium had to be cleaned. The cleaning procedure includes 3-step washing in ultrasonic bath cleaner (US) in (i) detergent, (ii) acetone (iii) ethanol, and subsequent boiling in MilliQ $\mathrm{H}_{2} \mathrm{O}$.

Electron beam evaporation (EBE) for the titanium deposition was performed in a modified Varian 3117 chamber, where parameters under control are given in Table 1. Starting material was 99\% Ti 0.2$1.5 \mathrm{~mm}$ granulate (Balzers, USA). The substrates were positioned onto a rotating calotte to ensure a uniform thickness of the layers over the sample surface. The substrates were preheated to $230^{\circ} \mathrm{C}$. Ti layers with targeted mass thickness $\sim 800 \mathrm{~nm}$ were deposited. Mass thickness of the layers was controlled by a quartz crystal monitor. Base pressure was $6.6 \cdot 10^{-4} \mathrm{~Pa}$.

Titanium layers $\sim 800 \mathrm{~nm}$ thick were deposited at room temperature by DC magnetron sputtering (MS) (60W power) on TCO coated glass substrates. Working gas was argon, target to substrate distance was $140 \mathrm{~mm}$. Base chamber pressure was $10^{-5} \mathrm{~Pa}$ and working gas pressure during deposition was $0.5 \mathrm{~Pa}($ Table 1$)$.

According to TCO and deposition technique, surfaces were denominated as electron beam evaporated titanium on AZO; E1, E2 and E3, while magnetron sputtered on AZO; M4, M5 and M6. Subsequently, magnetron sputtered Ti on AZO, ITO and FTO were denominated as MAZO, MITO and MFTO, respectively (Table 1).

The anodization was carried out at room temperature in a conventional two-electrode cell using a direct current (DC) power supply. A Pt foil was used as the counter electrode and a deposited Ti layer served as the anode. The electrolyte was $0.9 \mathrm{wt} \%$ ammonium fluorite $\left(\mathrm{NH}_{4} \mathrm{~F}\right.$, p.a. Kemika, Croatia) and $2 \mathrm{wt} \%$ milliQ $\mathrm{H}_{2} \mathrm{O}$ in ethylene glycol $\left(\mathrm{C}_{2} \mathrm{H}_{6} \mathrm{O}_{2}\right.$, p.a. Kemika, Croatia). A radial surface having diameter of $1 \mathrm{~cm}$ was exposed to the anodization; until mostly transparent.

The formation of a nanoporous structure during the anodization can be summarised as the result of three simultaneous processes: (1) field assisted oxidation of $\mathrm{Ti}$ and formation of $\mathrm{TiO}_{2}$, (2) field 
assisted oxide dissolution (dissolution of $\mathrm{Ti}^{4+}$ in the solution) and (3) chemical dissolution of $\mathrm{Ti}$ and $\mathrm{TiO}_{2}$, due to etching by fluoride ions $[43,44]$. At first, due to the applied electrical field, metal cations are formed (Eq. 1) and afterwards a compact oxide is created on the metal/electrolyte interface in the reaction with oxygen anion from water (Eq. 2).

$$
\begin{aligned}
& \mathrm{Ti} \rightarrow \mathrm{Ti}^{4+}+4 \mathrm{e}^{-} \\
& \mathrm{Ti}^{4+}+2 \mathrm{H}_{2} \mathrm{O} \rightarrow \mathrm{TiO}_{2}+4 \mathrm{H}^{+}
\end{aligned}
$$

Since the system is under constant voltage, the applied field enables transport of ions through the oxide lattice. Further oxide growth occurs on metal/oxide interface owing to the field assisted transport of $\mathrm{O}^{2-}$ downwards and on the oxide/electrolyte interface due to the transport of $\mathrm{Ti}^{4+}$ upwards [19]. In presence of the $\mathrm{F}^{-}$the oxide growth becomes less straight-forward as $\mathrm{TiO}_{2}$ chemically dissolves. During that process, soluble $\left[\mathrm{TiF}_{6}\right]^{2-}$ complex is produced (Eq. 3). It is possible that the reaction occurs at oxide/electrolyte as well at metal/oxide interface. Direct complexation (Eq. 4) at the oxide/electrolyte interface is also a possible way of $\left[\mathrm{TiF}_{6}\right]^{2-}$ formation due to $\mathrm{Ti}^{4+}$ transport.

$$
\begin{aligned}
& \mathrm{TiO}_{2}+6 \mathrm{HF} \rightarrow\left[\mathrm{TiF}_{6}\right]^{2-}+2 \mathrm{H}_{2} \mathrm{O}+2 \mathrm{H}^{+} \\
& \mathrm{Ti}^{4+}+6 \mathrm{~F}^{-} \rightarrow\left[\mathrm{TiF}_{6}\right]^{2-}
\end{aligned}
$$

In addition, on the oxide/electrolyte interface hydroxides or oxyhydroxides $\left(\mathrm{TiO}(\mathrm{OH})_{2}\right)[45,46]$ can be formed (Eq. 5) in the reaction with water or hydroxide ion (available from the hydrolysis reaction of $\mathrm{F}^{-}$). Simultaneously, at the cathode hydrogen evolution takes place (Eq. 6).

$$
\begin{aligned}
& \mathrm{Ti}^{4+}+(2+\mathrm{n}) \mathrm{H}_{2} \mathrm{O} \rightarrow \mathrm{TiO}_{2-\mathrm{n}}(\mathrm{OH})_{2 \mathrm{n}}+4 \mathrm{H}^{+} \\
& 2 \mathrm{H}_{2} \mathrm{O}+2 \mathrm{e}^{-} \rightarrow \mathrm{H}_{2}+2 \mathrm{OH}^{-}
\end{aligned}
$$

The final porous oxide layer is formed as a result of the continuous process, which involves competition between chemical dissolution of oxide and anodic oxide formation [24]. The $\mathrm{TiO}_{2}$ nanotube arrays were fabricated at $30 \mathrm{~V}$. Anodization parameters are given in Table 2. For the whole time of anodization, current was monitored and recorded in the form of I-t graphs. After anodization, the specimens were cleaned using isopropyl alcohol (IPA, $\left(\mathrm{C}_{3} \mathrm{H}_{8} \mathrm{O}\right.$, p.a. Kemika, Croatia) and MilliQ $\mathrm{H}_{2} \mathrm{O}$ treatment in UZV bath and dried in a nitrogen gas stream. Anodized samples were thermally 
treated at $450^{\circ} \mathrm{C}$ for 1 hour, in order to transform from amorphous to crystalline $\mathrm{TiO}_{2} \mathrm{NT}$, the heating and cooling was performed at the rate of $1{ }^{\circ} \mathrm{C} / \mathrm{min}$.

According to previous surfaces denomination, anodized and thermally treated nanostructured samples were denominated as E1A, E2A, E3A, M4A, M5A and M6A. NT samples on AZO, ITO and FTO were denominated as MAZOA, MITOA and MFTOA, respectively (Table 2).

\subsection{Characterisation techniques}

Morphology of the surfaces was investigated using field emission gun scanning electron microscopy (FEG-SEM) device JEOL model 7000F equipped by energy dispersive spectroscopy (EDS) system. Micrographs were analysed using fast Fourier transformation algorithm (FFT) in order to achieve information on the periodically repeating features and their dimensions.

For the scanning electron microscopy cross section of the $\mathrm{TiO}_{2}$ nanotubes arrays at different substrates, samples were prepared by using focused ion beam scanning electron microscopy system (FIB-SEM) FEI Helios Nanolab. Pt protective strap was deposited on the area of interest. The specimens were milled using $30 \mathrm{keV} \mathrm{Ga}$ ions to obtain the cross section area of interest.

The powder X-ray diffraction (XRD) was accomplished using Shimadzu diffractometer XRD 6000 with $\mathrm{CuK} \alpha$ radiation at acceleration voltage of $40 \mathrm{kV}$ and current of $30 \mathrm{~mA}$. Data were collected between $20-75^{\circ} 2 \theta$ in a step scan mode with steps of $0.02^{\circ} 2 \theta$ and counting time of $0.6 \mathrm{~s}$. Grazing indecent XRD (GI XRD) measurements were performed on Bruker ASX D8 Discover device 20$75^{\circ} 2 \theta$ in a step scan mode with steps of $0.01^{\circ}$ and counting time of $0.1 \mathrm{~s}$ at angle of $1.5^{\circ} \theta$ at acceleration voltage of $40 \mathrm{kV}$ and current of $40 \mathrm{~mA}$. The average crystallite size of titanium was calculated from the broadening of several diffraction peak using Scherrer's equation: $D=k \lambda(B \cos \theta)$, where $\mathrm{D}$ is the average crystallite diameter, $\mathrm{k}$ is Scherrer's constant, $\lambda$ is the $\mathrm{X}$-ray wavelength, $\mathrm{B}$ is full width at the half height of the diffraction peak corrected for instrumental broadening, and $\theta$ is the diffraction angle. XRD scans embed also the horizontal surfaces beyond the boundaries of anodized region. 
The micro-Raman spectroscopy measurements were performed using a Horiba Jobin-Yvon T64000 system equipped with a Coherent, Innova 400 argon ion laser operating at $514.5 \mathrm{~nm}$ for the excitation. The laser power of $20 \mathrm{~mW}$ was focused on the samples using a $50 \times$ magnification distance objective.

The UV-VIS spectrum of the prepared samples were obtained using Perkin Elmer Lambda 900 in transmission mode. The spectra were recorded at room temperature in the wavelength range $200-800$ $\mathrm{nm}$ with step of $1 \mathrm{~nm}$ and integrating time of $0.8 \mathrm{~s}$.

\section{Results and discussion}

\subsection{Titania based solar cell prerequisites}

It has to be ensured that the glass substrate and the transparent conductive layer are perfectly uniform and clean, only then may they be excluded as the process parameter. Then the parameters of the deposition process may be held responsible for the differences in nanostructured products, assuming all anodization parameters were held constant [47]. This is necessary to ensure good adhesion of deposited layer. Non-inverted setup, which requires Ti deposition on TCO, seems to be more appropriate. Methods applied to deposit titanium on equally prepared glass substrates with TCO layer mainly differ on applied energy; magnetron sputtering was used as a high energy deposition method, while electron beam evaporation was used as a low energy deposition method $[48,49]$. Both deposition methods were performed under just marginally different conditions.

\subsection{Anodized nanostrutrured titania layer properties}

Scanning electron microscopy micrographs of all anodized titanium layers are presented on Figure 2. One can observe that several nanostructural features arise; tubes, porous structures, wormholes, and comb-like blend of all the mentioned morphologies. Among the plethora of samples, only those having distinctively different morphologies were selected; 3 for EBE derived (Figure 2a,b,c), and 3 for MS derived (Figure 2d,e,f) (additional info in Supplementary material). However, it is important to bear in mind that the selected morphologies do not relate to their abundance. The 
favourable products are the nanotubes (Figure 2a,d), while the formation of other type of nanostructures is not favourable (Figure $2 b$ ). We observed a variety of inhomogeneous surfaces, where wormhole-like structures with different content of tubes were formed (Figure 2c,e,f). The best samples display uniformly distributed, equally shaped self-standing nanotubes (Figure 2a,d). Based on the anodization voltage, the tubes displayed the inner tube diameter in range from 20-35 nm, and outer in range 40-70 nm, which was confirmed using FFT analysis of the micrographs. FFT analysis of sample M4A (Figure 2d) was shown in Figure 3. The authors were successful in preparation of favourable NT in lengths from 200 up to $2000 \mathrm{~nm}$, yet mainly lengths around $800 \mathrm{~nm}$ were prepared, as observed from SEM micrographs side view (shown in Figure 4 and in Supplementary material). NT in all of these lengths should be acceptable for DSSC. For PSC, the success of infiltration of active material decreases with NT depth, so average length of tubes of $800 \mathrm{~nm}$ should be appropriate. Upon thermal treatment, the nanotubes were confirmed as anatase using Raman spectroscopy ( $E_{\mathrm{g}}$ modes at 639, 197 and $144 \mathrm{~cm}^{-1}, \mathrm{~A}_{1 \mathrm{~g}} / \mathrm{B}_{1 \mathrm{~g}}$ modes at $516 \mathrm{~cm}^{-1}$ and $\mathrm{B}_{1 \mathrm{~g}}$ mode at $399 \mathrm{~cm}^{-1}$ ) and GI XRD (ICDD PDF\#211272) (Figure 5ab) [50]. The interpretation is focused on the microscopic features of the nanostructures, while the macroscopical overview of the samples may reveal additional aspects that may significantly contribute to the applicability of the samples in the solar cell device. The desirable sample characteristic would be a large scale homogeneity and uniformity, and absence of all defects and cracks. Some properties of the starting titanium layer may also influence the macroscopic features of the titania nanotube layer. This will be commented later. Also, some samples show debris on top of the titania ordered nanotubes, whereas the EDS chemical composition suggests this material is also titania. This material is only superficially adsorbed to the surface and can be removed using ultrasonic tip. For all samples where such effect was evidenced, the debris layer was removed by treatment in US bath.

\subsection{Titanium layer properties}

\subsubsection{Structural parameters}

The nanotubes were assigned as anatase using GI XRD (Figure 5b). Titanium occurs also as the area where diffraction signal was collected extend beyond the anodized circle. 
More attention was dedicated to the as-deposited starting layers; all titanium samples show similar diffractograms (Figure 6b). Major feature is the zincite (ICDD PDF\#36-1451). It is common for zincite thin films to have quite strong [002] reflection due to a preferred orientation which occurs for all measured samples. Titanium phase is visible in all samples and it is assigned as $\alpha$-titanium hexagonal close packing (hcp) (ICDD PDF\#44-1294). Beside the $\alpha$-titanium, there might be a presence of body centred cubic (bcc) $\beta$-titanium phase (ICDD PDF\#88-2321). No additional phases were present. Even though the conditions for stable growth of $\beta$-titanium phase were not reached, local existence of $\beta$-titanium is not excluded. The more the peak intensity in the diffractograms differs from $\alpha$-titanium ICDD data in terms of both preferred orientation or possible $\beta$-phase existence, the less favourable thin film microstructure turns out to be. It was observed that titanium thin films also show a strong preferred orientation, again on behalf of the reflection [002]. Previous investigation shows that the more power is employed during the deposition, the derived titanium particles turn out to have a stronger preferred orientation [51], while further increase in power will yield surface porosity. We observed that different deposition methods also strongly favour different preferred orientation (at different planes), while other conditions determinate if the achieved texture will be favourable. It could be said that for the deposition at lower temperatures the strain remains in samples. Namely, some shifts in [002] position point out that the interplanar distances of the unit cell are affected by different deposition conditions. Similar effects were observed by Chawla et. al. [52] for different levels of energy during the deposition. This all may be attributed to the fact that the different surface free energies are associated with different planes. One should not forget that different materials with different thermal expansion coefficients are interfaced. Obviously, there is a presence of competition between deposition energy and surface free energy affecting the textures of the grains, which altogether heavily depends on the different deposition parameters, all of which are related with substrate processing temperature, pressure, power and other substrate properties in complex manner [52-56]. The non-isotropic particles, i.e. the 1D or 2D growth of crystals may be the main cause for such result. Therefore, the normalised relative ratios of the selected reflections [002] and [103] to the ICDD card data were plotted (Figure 7a). Values significantly different than 1 (especially bigger values) suggest presence of non-isotropic crystallites and strain. It could be concluded that successful 
nanotubes will be derived from the titanium thin film consisting of mainly isotropic particles. Limited number of titanium peaks hinders the deeper structural insight in these presumptions, especially in the case of Ti deposited by magnetron sputtering where this quantification is not reasonable for all samples. One can only assume higher energy method will cause higher extent of preferred orientation. Literature also strongly relates the degree of orientation on the texture and roughness of underlying material [57], whereas magnetron sputtered Ti thin films generally show [100], [103], and [101] crystallographic orientations of crystallites parallel to the surface. Only a special modification of the magnetron sputtering process (closed-field unbalanced, pulsed), can bring up the number of various and independent deposition parameters, i.e. can enable optimizing of deposited layers [58]. Influences of underlaying surface and deposition conditions will be discussed later. Based on the diffraction parameters, the average crystallite sizes were calculated; results are shown in Figure $7 \mathrm{~b}$. The sizes range from 40-180 $\mathrm{nm}$. It was observed that smaller crystallites of used deposited titanium lead to more successful nanotubes, as will be shown later. The particle size is usually determined by the temperature of the deposition, yet this investigation shows that other parameters can also be responsible for the grain size change.

\subsubsection{Microstructural parameters}

In order to confirm the structural results, morphologies of titanium layers deposited by electron beam evaporation and magnetron sputtering were investigated with FEG-SEM; (Fig. 7). Different morphologies were observed even though the deposition parameters differed minimally (Table 1). Several types of surfaces can be observed; dense, almost absent of pores, homogeneous with narrow size distribution of isotropic particles (E1) (Fig. 7a), less dense with 2D flakes and irregular particles of roughly the same size that form significant amount of pores and holes (E2) (Fig. 7b), less dense surface consisting of 2D objects, flakes and 1D pillars, agglomerated, in brush style, again with lot of porosity (E3) (Fig. 7c), dense surface with expressed roughness, almost absent of pores, homogeneous with equally sized isotropic particles (M4) (Fig. 7d), less dense with randomly oriented 2D and irregular particles that form significant amount of pores and holes (M5) (Fig. 7e), dense with 2D particles that also display porous surface with holes (M6) (Fig. 7f). The results are in concordance 
with the XRD structural analysis; smaller and more uniform titanium particles yield more successful titania nanotubes. It seems that titanium surface with 1D, 2D, generally non-isotropic, non-uniformly size distributed and generally larger particles will allow excess etching that will prevent formation of a homogeneous, ordered titania nanotube layer at a microscale, and without defects and cracks at a macroscale. Only the dense homogeneous titanium layer surface, uniform in thickness at macroscale, having uniformly seized isotropic nanoparticles will enable uniform etching and thus favourable reproducible formation of the nanotubes. The results enable us to offer relation of the achieved titanium morphology type to the type of morphology and self-organisation of the derived anatase tubes at the nano level. Titanium-to-titania (titanium morphology leading to titania nanotubes formation) relation was summarised on the scheme in Figure 8.

Successful NT layer was achieved at the titanium surface (sample E1) using electron beam evaporation at base pressure of $6.6 \cdot 10^{-4} \mathrm{~Pa}$, deposition rate of $8.0 \mathrm{As}^{-1}$ on substrates preheated at $230^{\circ} \mathrm{C}$ (Table 1). Successful NT layer was achieved at the titanium surface (sample M4) prepared using magnetron sputtering at starting pressure of $10^{-5} \mathrm{~Pa}$, working Ar pressure of $0.5 \mathrm{~Pa}$ and deposition power of $60 \mathrm{~W}$ at room temperature (Table 1). Generally, somewhat more homogeneous surfaces appear to be achieved by magnetron sputtering. However, it can be observed that even the surfaces that appear to be similar (in terms of microstructural homogeneity) (Figure 9d,e,f) do not always yield same nanotubes (Figure 2d,e,f). A different level of titanium particles adhesion is achieved using different deposition methods, whereas magnetron sputtering, as a higher energy deposition method, obviously exhibits the surfaces where somewhat stronger bonds are achieved. Although magnetron sputtering should be nominated as the method of choice based on this theoretical consideration, equally favourable NT can be prepared at titanium thin films deposited by electron beam evaporation.

\subsubsection{Electrochemical etching conditions}

The course of the anodization process which leads to formation of different morphologies may be monitored by anodization currents (Figure 10a). Contrary to general belief, very stable decay of current will not yield ideal nanotubes, only the porous surface. That may be related to the mechanisms of etching, which occur in a stepwise manner. So, the abrupt changes in current obviously point out to 
local resistivity increase as nonconductive titania layer is formed and the resistivity decreases when nanotubes form. The electric mechanism of reaction is not offered. However, empirically it was observed that, after the starting current decay, sudden current increase may be a good indication that NT are formed. Additionally, very "noisy" current decay may present the new NT formation and thus is more favourable than very stable decay. Absence of the current increase in the later stage of anodization may prevent the penetration of the layers, i.e. excess etching. I-t curves can serve only as marginal parameter in the anodization process; they are hardly a reliable indicator that the electrochemical etching process is executed in concise and homogeneous manner and offer little information on the type of morphology and organisation of the $\mathrm{TiO}_{2}$ NT achieved. Probably the I-t effects arise as a consequence of macroscopical issues on the treated surfaces as well.

The macroscopical issues observed on micrographs of the nanostructured titania (Figure 2a) may also originate from the properties of the titanium layer. For example, it was observed that higher surface roughness of the titanium layer will in turn give more cracks in the nanotube layer. However, majority of the cracks seem to be caused by the contamination particles. Based on that and the lack of statistics on these observations, the macroscopical effects on the nanotube surface should not be related solely to the starting titanium layer parameters.

For the photovoltaic device application, it is important to achieve uniform etching to the titania nanotubes. Namely, insufficient etching will cause the leftovers of titanium layer. This will lead to reduction or complete lack of transparency required for the solar cell to work. Alternatively, the excess etching will dissolve the entire layer trough to the TCO layer (Figure 10b). This will cause the short circuit and the solar cell will not work. Optimisation of deposition requires the titanium layer with uniform and known thickness all over the sample. Lengths of anodized $\mathrm{TIO}_{2} \mathrm{NT}$ usually correspond to Ti thin-film thickness, whereas under some minimum Ti thin-film thickness value the NT will not be formed. The use of organic viscous electrolytes may allow additive growth, yielding NT that surpasses the thickness of the consumed Ti layer several times [56,59]. In our case, the Ti layer thickness was approx. $0.8 \mu \mathrm{m}$ which was reported as sufficient for the NT [60,51]. Following the acceptable Ti layer, in the next step the duration of the anodization (with all other parameter constant) has to be optimised 
in order to yield (in ideal case) complete anodization of the titanium layer without penetration into the TCO layer. In practice, titanium layer leftover with thickness of several nanometers will ensure reasonable titania nanotube layer with sufficient transparency. The thermal treatment to anatase will further increase the transparency to more than $80 \%$ (UV-VIS spectra shown in Figure 10c). Of course the better the NT morphology turns out to be, the more transparent are the layers, while for all samples the bandgap is only marginally affected.

\subsubsection{Deposition conditions}

Is it possible to exactly define the ideal conditions to derive titanium that would yield continuous and uniform layer of titania nanotubes?

For the evaporation method, the ideal conditions would be those that yield smaller, homogeneous isotropic particles. Namely, the electron beam evaporation is low energy deposition method, meaning the particle density in the deposited layer will not be very high (thus the adhesion between the particles will not be very strong). Therefore, much will depend on the extent of the sites favourable for etching. When big particles are in contact, big voids are enabled. Same applies for the non-isotropic particles. The electron beam evaporation conditions have complex mutual dependency. However, high deposition rate without substrate preheating seems to be more favourable than the low deposition rate with substrate preheating.

The magnetron deposition conditions seem to have less influence on the nanotubes outcome and more influence on the general layer properties. Magnetron sputtering is a high energy deposition method, meaning the particle density in the deposited layer will be high, i.e. all the deposited particles show strong adhesion, so voids leading to excess etching will be reachable less often. However, the issue here is the presence of cracks in the derived nanotubes layer. This may be due to the difference in thermal expansion coefficients of TCO and titanium. Usually very thick or very thin layers show more favourable behaviour but that somewhat limits the availability of the nanotube lengths. Although the magnetron sputtering should be the method of choice based on these theoretic considerations, there are still no conclusive practical arguments to backup these claims. For Ti thin film deposition, it was observed that the substrate temperature during the process strongly influences the quality of the thin 
film and its adhesion to the substrate, which are crucial for obtaining free standing NT on TCO. Low temperatures of substrate heating yield poor connection of Ti and the substrate (whether because of poor adhesion or high residual stress), which will cause problems in subsequent anodization due to irregular film etching or peeling off. The exact reason for this effect is not clear, but it has been reported that the size of $\mathrm{Ti}$ crystalline grains is reversely proportional to the temperature of the substrate during sputtering, although it is not apparent if it directly influences NT growth mechanism and morphology $[61,62,18,56]$. To enhance Ti layer density and grain size, an increase in sputtering substrate temperature or subsequent thermal treatment may be suggested, and in order to influence the dimensionality of achieved particles the deposition power optimisation may be suggested $[48,49]$. However, the temperature increase inevitably enlarges grain size which was found as unfavourable. It is better to say that smaller particles are more favourable for successful NT growth, but only when good layer density and adhesion of Ti layer is achieved (with constant strain and preferred orientation) despite the deposition method employed. Practically, the difference in samples may arise as the distinctive deposition session exhibit marginal differences due to minor pressure corrections, cooling conditions, cathode material wear, etc. Overall, this investigation shows more parameters lay behind the control of the deposition of the Ti thin-films exhibiting appropriate morphology for appropriate etching susceptibility.

\subsection{Influence of TCO on CTO}

Previously it was mentioned that underlaying material may significantly influence the parameters of the overlaying [57]. In order to check if the influence on titania nanotubes may be somehow related to the type of the material used for TCO layer, subsequently the NT samples were prepared on different transparent conductive oxides (aluminium zinc oxide (AZO), fluorine tin oxide (FTO) and indium tin oxide (ITO)). By relating XRD (Figure 11) and SEM (Figure 12abc) data, it was observed that titanium morphology and structure derived on these layers show very little differences. XRD shows well defined structures with crystallite sizes ranging from $46-57 \mathrm{~nm}$, which was previously determined as desirable. Morphologies observed in FEG-SEM micrographs, in general resemble to the description of surfaces previously shown to be favourable for the growth of the NT by anodization. 
However, deeper insight reveals one important deviation from the ideal morphology; it is the size of the Ti particles or the particle agglomerates, i.e. surface roughness. Anodized nanotubes (Figure 12def) also do not significantly differ, but follow the previously established rule where smaller $\mathrm{Ti}$ particles yield better NT. Thus, the deposition is responsible for the achieved titanium morphology (and structurally preferred morphology) and influence on anodization, while the boundary to TCO layer has less influence on subsequent layers, i.e. titanium layer and nanotubes anodized thereof. One should not neglect the fact that the TCO surface morphology may be transferred on the Ti surface morphology. Thereby the use of FTO (typically showing surface constituted of large particles or more agglomerated particles or higher surface roughness) may be less favourable than the AZO or ITO (typically showing fine particles and low surface roughness). Authors observe lower success rate (not statistically analysed). Best samples containing glass substrate/TCO/Ti/anatase nanotubes/ should be favourable for the DSSC or PSC solar cell assembly.

\section{Conclusions}

The prepared titanium samples yield some difference in surface morphologies. Based on that, samples having different morphologies were also found to exhibit different susceptibility to successful and reproducible anodization. Differences in the growth of the $\mathrm{TiO}_{2} \mathrm{NT}$ with constant main anodization parameters were attributed to different properties of the starting titanium layer.

It was found that the morphology of the starting titanium surface had significantly determined the nanostructure formation. For advanced application, precise control of properties (and the deposition properties) of these thin films is required. It was determined that all key factors of the starting layer preparation; control of microstructure, surface condition and geometry, equally contribute to derived titania nanotube properties. Once precise control of the parameters of titanium thin film deposited on the TCO layer is achieved, the TCO layers will have less effect on the $\mathrm{TiO}_{2} \mathrm{NT}$ derived from the titanium layer. 
It was determined that the deposited titanium has to be uniform in thickness, with as small as possible nanocrystals. Most important, the nanoparticles should be distributed homogeneously and as isotropic as possible. Only then the anodization process will be executed in a liner manner. It seems that magnetron sputtering may offer better deposition conditions.

There is no unique recipe suggestion for the anatase nanotube production, only general upgrade suggestions. For the upgrade in undesired macroscopical issues, only general laboratory practice may offer benefits. It is expected that the best samples containing glass-substrate/AZO-thin-film/Ti-thinfilm/anatase nanotubes/etc. layers are favourable for the DSSC and PSC solar cell assembly.

\section{Acknowledgement}

The financial support of the ESF project No HR.3.2.01-0312 "Titania nanotubes for photovoltaic application", COST MP 1307 STSM project No 32084 "The electric compatibility of TCO and CT layers in DSSC and OPV solar cells" and CSF project No 9419 "Zinc Oxide and Titanium Dioxide Nanocomposites for Photovoltaic Application” are gratefully acknowledged.

\section{References}

1. A.R. Jordeh, Parameter estimation of solar photovoltaic (PV) cells: A review, Renew. Sustain. Energy Rev. 61 (2016) 354-371.

2. Y. Du, H. Cai, H. Wen, Y. Wu, L. Huang, J. Ni, J. Li, J. Zhang, Novel Combination of Efficient Perovskite Solar Cells with Low Temperature Processed Compact $\mathrm{TiO}_{2}$ Layer Via Anodic Oxidation, ACS Appl. Mater. Interfaces 8 (2016) 12836-12842.

3. Y. Huang, J. Wu, D. Gao, High-efficiency perovskite solar cells based on anatase $\mathrm{TiO}_{2}$ nanotube arrays, Thin Solid Films 598 (2016) 1-5.

4. X. Chen, S.S. Mao, Titanium dioxide nanomaterials: synthesis, properties, modifications, and applications. Chem Rev. 107 (2007) 2891-2959. 
5. J. Gong, J. Liang, K. Sumathy, Review on dye-sensitized solar cells (DSSC): Fundamental concepts and novel materials, Renew. Sustain. Energy Rev. 16 (2012) 5848-5860.

6. A. Kongkanand, K. Tvrdy, K. Takechi, M. Kuno, P.V. Kamat, Tuning photoresponse through size and shape control of CdSe- $\mathrm{TiO}_{2}$ architecture, J. Am. Chem. Soc. 130 (2008) 4007-4015.

7. K. Zhu, N.R. Neale, A. Miedaner, A.J. Frank, Enhanced charge-collection efficiencies and light scattering in dye-sensitized solar cells using oriented $\mathrm{TiO}_{2}$ nanotubes arrays, Nano Lett. 7 (2007) $69-74$.

8. C.C. Raj, R. Prasanth, A critical review of recent developments in nanomaterials for photoelectrodes in dye sensitized solar cells, J. Power Sources 317 (2016) 120-132.

9. M.A. Green, K. Emery, Y. Hishikawa, W. Warta, E.D. Dunlop, Solar cell efficiency tables (version 44), Prog. Photovolt. Res. Appl. 22 (2014) 701-710.

10. S. Mathew, A. Yella, P. Gao, R.H. Baker, B.F.E. Curchod, N.A. Astani, I. Tavernelli, U. Rothlisberger, M.K. Nazeeruddin, M. Gratzel, Synthesis, characterization and ab initio investigation of a panchromatic ullazine-porphyrin photosensitizer for dye-sensitized solar cells, Nat. Chem. 6 (2014) 242-247.

11. J.A. Seabold, K. Shankar, R.H.T. Wilke, M. Paulose, O.K. Varghese, C.A. Grimes, K.S. Choi, Photoelectrochemical properties of heterojunction $\mathrm{CdTe} / \mathrm{TiO}_{2}$ electrodes constructed using highly ordered $\mathrm{TiO}_{2}$ nanotube arrays, Chem. Mater. 20 (2008) 5266-5273.

12. J. Weickert, C. Palumbiny, M. Nedelcu, T. Bein, L. Schmidt-Mende, Controlled growth of $\mathrm{TiO}_{2}$ nanotubes on conducting glass, Chem. Mater. 23 (2011) 155-162.

13. G. Butail, P.G. Ganesa, M. Raddiar, R. Teki, N. Ravishankar, D.J. Dudquette, G. Ramanath, Kinetics of titania nanotube formation by anodization of titanium films, Thin Solid Films 519 (2011) 1821-1824.

14. T. Stergiopoulos, A. Valota, V. Likodimos, Th. Speliotis, D.Niarchos, P. Skeldon, G.E. Thompson, P. Falaras, Dye-sensitization of self-assembled titania nanotubes prepared by galvanostatic anodization of Ti sputtered on conductive glass, Nanotechnology 20 (2009) $365601-365610$. 
15. Y. Tang, J. Tao, Z. Dong, J.T. Oh, Z. Chen, $\mathrm{TiO}_{2}$ nanotube arrays by anodization of titanium film on conducting glass substrate, Adv. Nat Sci.: Nanosci. Nanotechnol. 2 (2011) 045002-045008.

16. H. Zheng, A.Z. Sadek, M. Breedon, D. Yao, K. Latham, J. du Plessis, K.K. Zadeh, Fast formation of thick and transparent titania nanotubular films from sputtered Ti, Electrochem. Commun. 11 (2009) 1308-1311.

17. J. Qiu, F. Zhuge, K. Lou, X. Li, X. Gao, X. Gan, W. Yu, H.K. Kim, Y.H. Hwang, A facile route to aligned $\mathrm{TiO}_{2}$ nanotube arrays on transparent conducting oxide substrates for dye-sensitized solar cells, J. Mater. Chem. 21 (2011) 5062-5068.

18. J.Y. Kim, J.H. Noh, K. Zhu, A.F. Halverson, N.R. Neale, S. Park, K.S. Hong, A.J. Frank, General strategy for fabricating transparent $\mathrm{TiO}_{2}$ nanotube arrays for dye-sensitized photoelectrodes: Illumination geometry and transport properties, ACS Nano 4 (2011) 2647-2656.

19. J.M. Macak, H. Tsuchiya, A. Ghicov, K. Yasuda, R. Hahn, S. Bauer, P. Schmuki, TiO 2 nanotubes: self-organized electrochemical formation, properties and applications, Curr. Opin. Solid State Mater. Sci. 11 (2007) 3-18.

20. H. Tsuchiya, J.M. Macak, L. Taveira, E. Balaur, A. Ghicov, K. Sirotna, P. Schmuki, Selforganized $\mathrm{TiO}_{2}$ nanotubes prepared in ammonium fluoride containing acetic acid electrolytes, Electrochem. Commun. 7 (2005) 576-580.

21. S. Li, G. Zhang, D. Guo, L. Yu, W. Zhang, Anodization fabrication of highly ordered $\mathrm{TiO}_{2}$ nanotubes, J. Phys. Chem. C 113 (2009) 12759-12765.

22. P. Roy, S. Berger, P. Schmuki, $\mathrm{TiO}_{2}$ nanotubes: Synthesis and Application, Angew. Chem. Int. Ed. Engl. 50 (2011) 2904-2939.

23. V. Zwilling, M. Aucouturie, E. Darque-Ceretti, Anodic oxidation of titanium and TA6V alloy in chromic media. An electrochemical approach, Electrochim. Acta 45 (1999) 921-909.

24. A. Ghicov, P. Schmuki, Self-ordering electrochemistry: a review on growth and functionality of $\mathrm{TiO}_{2}$ nanotubes and other self-aligned MOx structures, Chem. Commun. 20 (2009) 2791-2808.

25. S. Bauer, S. Kleber. P. Schmuki, $\mathrm{TiO}_{2}$ nanotubes: Tailoring the geometry in $\mathrm{H}_{3} \mathrm{PO}_{4} / \mathrm{HF}$ electrolytes, Electrochem. Commun. 8 (2006) 1321-1325. 
26. J. M. Macak, H. Tsuchiya, P. Schmuki, High-aspect-ratio $\mathrm{TiO}_{2}$ nanotubes by anodization of titanium, Angew. Chem.-Int. Edit. 44 (2005) 2100-2102.

27. S.P. Albu, A. Ghicov, J.M. Macak, P. Schmuki, $250 \mu \mathrm{m}$ long anodic $\mathrm{TiO}_{2}$ nanotubes with hexagonal self-ordering, Phys. Status Solidi-Rapid Res. Lett. 1 (2007) R65-R67.

28. M. Paulose, H.E. Prakasam, O.K. Varghese, L. Peng, K.C. Popat, G.K. Mor, T.A. Desai, C.A. Grimes, $\mathrm{TiO}_{2}$ Nanotube Arrays of $1000 \mu \mathrm{m}$ Length by Anodization of Titanium Foil: Phenol Red Diffusion, J. Phys. Chem. C 111 (2007) 14992-14997.

29. G. Ali, C. Chen, S.H. Yoo, J.M. Kum, S.O. Cho, Fabrication of complete titania nanoporous structures via electrochemical anodization of Ti, Nanoscale Res. Lett. 6 (2011) 332.

30. L. Taveira, A. Sagues, J.M. Macak, P. Schmuki, Impedance behavior of $\mathrm{TiO}_{2}$ nanotubes formed by anodization in NaF electrolytes, J. Electrochem. Soc. 155 (2008) C293-C302.

31. L. Sun, S. Zhang, X.W. Sun, X. He, Effect of electric field strength on the length of anodized titania nanotube arrays, J. Electroanal. Chem. 637 (2009) 6-12.

32. J.M. Macak, L.V. Taveira, H. Tsuchiya, K. Sirotna, J. Macak, P. Schmuki, Influence of different fluoride containing electrolytes on the formation of self-organized titania nanotubes by $\mathrm{Ti}$ anodization, J. Electroceram. 16 (2006) 29-34.

33. C. Ruan, M. Paulose, O.K. Varghese, G.K. Mor, C.A. Grimes, Fabrication of highly ordered $\mathrm{TiO}_{2}$ nanotube arrays using an organic electrolyte, J. Phys. Chem. B 109 (2005) 15754-15759

34. J. Wang, Z. Lin, Anodic Formation of Ordered $\mathrm{TiO}_{2}$ Nanotube Arrays: Effects of Electrolyte Temperature and Anodization Potential, J. Phys. Chem. C 113 (2009) 4026-4030.

35. V.M. Prida, E. Manova, V. Vega, M. Hernandez-Velez, P. Aranda, K.R. Pirota, M. Vazquez, E. Ruiz-Hitzky, Temperature influence on the anodic growth of self-aligned Titanium dioxide nanotube arrays, J. Magn. Magn. Mater. 316 (2007) 110-113.

36. F.M.B. Hassan, H. Nanjo, M. Kanakubo, I. Ishikawa, M. Nishioka, Effect of Ultrasonic Waves on the Formation of $\mathrm{TiO}_{2}$ Nanotubes by Electrochemical Anodization of Titanium in Glycerol and $\mathrm{NH}_{4}$ F, e-J. Surf. Sci. Nanotech. 7 (2009) 84-88.

37. Z. Lockman, S. Sreekantan, S. Ismail, L. Schmidt-Mende, J.L. MacManus-Driscoll, Influence of anodisation voltage on the dimension of titania nanotubes, J. Alloys Compd. 503 (2010) 359-364. 
38. Y.V. Bhargava, Q.A.S. Nguyen, T.M. Devine, Mechanistic aspects of the self-organization process for oxide nanotube formation on valve metals, J. Electrochem. Soc. 156 (2009) E62-E68.

39. O.K. Varghese, M. Paulose, C.A. Grimes, Long vertically aligned titania nanotubes on transparent conducting oxide for high efficient solar cells, Nature Nanotechnol. 4 (2009) 592597.

40. S. Rani, S.C. Roy, M. Paulose, O.K. Varghese, G.K. Mor, S. Kim, S. Yoriya, T.J. LaTempa, C.A. Grimes, Synthesis and applications of electrochemically self-assembled titania nanotube arrays, Phys. Chem. Chem. Phys. 12 (2010) 2780-2800.

41. K. Shankar, J.I. Basham, N.K. Allam, O.K. Varghese, G.K. Mor, X.J. Feng, M. Paulose, J.A. Seabold, K.S. Choi, C.A. Grimes, Recent advances in the use of $\mathrm{TiO}_{2}$ nanotube and nanowire arrays for oxidative photoelectrochemistry, J. Phys. Chem. C 113 (2009) 6327-6359.

42. K. Siuzdak, M. Abbas, L. Vignau, M. Devynck, G. Dubacheva, A. Lisowska-Oleksiak, Application of non-metal doped titania for inverted polymer solar cells, J. Appl. Phys. 112 (2012) $123110-123114$.

43. G. E. Thompson, Porous anodic alumina: fabrication, characterization and applications, Thin Solid Films 297 (1997) 192-201.

44. G.K. Mor, O.K. Varghese, M. Paulose, N. Mukherjee, C.A. Grimes, Fabrication of tapered, conical-shaped titania nanotubes, J. Mater. Res. 18 (2003) 2588-2593.

45. L.V. Taveira, J.M. Macák, H. Tsuchiya, L.F.P. Dick, P. Schmuki, Initiation and Growth of SelfOrganized $\mathrm{TiO}_{2}$ Nanotubes Anodically Formed in $\mathrm{NH}_{4} \mathrm{~F} /(\mathrm{NH} 4)_{2} \mathrm{SO}_{4}$ Electrolytes, J. Electrochem. Soc. 152 (2005) B405-B410.

46. S.P. Albu, A. Ghicov, S. Aldabergenova, P. Drechsel, D. LeClere, G.E. Thompson, J.M. Macak, P. Schmuki, Formation of Double-Walled $\mathrm{TiO}_{2}$ Nanotubes and Robust Anatase Membranes, Adv. Mater. 20 (2008) 4135-4139.

47. T.H. Choudhury, S. Raghavan, Anodization of sputtered metallic films: The microstructural connection, Scripta Materialia 105 (2015) 18-21. 
48. Y. Jie, K. Lin, H. Zheng, C. Liu, L. Dudik, J. Zhu, C. Burda, Solar-light photoamperometric and photocatalytic properties of quasi-transparent $\mathrm{TiO}_{2}$ nanoporous thin films, ACS Appl. Mater. Inter. 2 (2010) 3075-3082.

49. M. Paulose, K. Shankar, O.K. Varghese, G.K. Mor, C.A. Grimes, Application of highly-ordered $\mathrm{TiO}_{2}$ nanotube arrays in heterojunction dye sensitized solar cells, J Phys D: Appl. Phys. 39 (2006) $2498-2503$

50. T. Ohsaka, F. Izumi and Y. Fujiki, Raman Spectrum of Anatase, $\mathrm{TiO}_{2}$, J. Raman spectrosc. 6 (1978) 321-324

51. Y. Tang, J. Tao, Y. Zhang, T. Wu, H. Tao, Y. Zhu, Preparation of $\mathrm{TiO}_{2}$ nanotube on glass by anodization of Ti films at room temperature, Trans. Nonferrous. Met. Soc. China 19 (2009) 192198.

52. V. Chawla, R. Jayaganthan, A.K. Chawla, R. Chandra, Microstructural Characterizations of Magnetron Sputtered Ti Films on Glass Substrate. J. Mater. Process. Tech. 209 (2009) 3444 3451.

53. Y.L Jeyachandran, B.Karunagaran, S.K. Narayandass, D. Mangalaraj, T.E. Jenkins, P.J. Martin, Properties of Titanium Thin Films Deposited by dc Magnetron Sputtering. Mat. Sci. Eng. AStruct, 431 (2006) 277-284.

54. T. Oya, E. Kusano, Effects of Radio-Frequency Plasma on Structure and Properties in Ti Film Deposition by dc and Pulsed dc Magnetron Sputtering, Thin Solid Films 517 (2009) 5837-5843.

55. P. Singh, D. Kaur, Influence of Film Thickness on Texture and Electrical Properties of Room Temperature Deposited Nanocrystalline $\mathrm{V}_{2} \mathrm{O}_{5}$ Thin Films. J. Appl. Phys. 103 (2008) 043507.

56. G.K. Mor, O.K. Varghese, M. Paulose, C.A. Grimes, Transparent Highly Ordered $\mathrm{TiO}_{2} \mathrm{Nanotube}$ Arrays via Anodization of Titanium Thin Films, Adv. Funct. Mater. 15 (2005) 1291-1296.

57. G.F. Iriarte, J. Bjurstrom, J. Westlinder, F. Engelmark, I.V. Katardjiev, Synthesis of c-axisoriented AlN thin films on high-conducting layers: Al, Mo, Ti, TiN, and Ni, IEEE T. Ultrason. Ferr. 52 (2005) 1170-1174. 
58. F.J. Jing, T.L. Yin, K. Yukimura, H. Sun, Y.X. Leng, N. Huang, Titanium film deposition by high-power impulse magnetron sputtering: influence of pulse duration, Vacuum 86 (2012) 2114 2119

59. S. Berger, J. Kunze, P. Schmuki, D. LeClere, A.T. Valota, P. Skeldon, G.E. Thompson, A lithographic approach to determine volume expansion factors during anodization: Using the example of initiation and growth of $\mathrm{TiO}_{2}$-nanotubes, Electrochim. Acta 54 (2009) 5942-5948.

60. S.L. Lim, Y. Liu, J. Li, E.T Kang, C.K. Ong, Transparent titania nanotubes of micrometer length prepared by anodization of titanium thin film deposited on ITO, Appl. Surf. Sci. 257 (2011) $6612-6617$.

61. A.Z. Sadek, H. Zheng, K. Latham, W. Wlodarski, K. Kalantar-Zadeh, Anodization of Ti Thin Film Deposited on ITO, Langmuir 25 (2009) 509-514.

62. H. Tsuchiya, T. Akaki, J. Nakata, D. Terada, N. Tsuji, Y. Koizumi, Y. Minamino, P. Schmuki, S. Fujimoto, Metallurgical aspects on the formation of self-organized anodic oxide nanotube layers, Electrochim. Acta 54 (2009) 5155-5162. 


\section{Captions}

\section{Figures}

Figure 1. Scheme of the anatase nanotubes preparation by titanium anodization.

Figure 2. Micrographs of the titania morphology and organisation forms derived by anodization of titanium thin films deposited by electron beam evaporation; a) sample E1A, b) sample E2A and c) sample E3A, and by magnetron sputtering; d) sample M4A, d) sample M5A and d) sample M6A.

Figure 3. Example of FFT micrograph analysis of the sample M4A (Figure 2d); dimensions of the periodical units, Insets: micrograph of analysed area of the sample M4A and FFT image of the micrograph.

Figure 4. Sideview micrographs of the NTs anodized from titanium thin films deposited by magnetron sputtering on top of a) AZO, b) FTO and c) ITO; additionally available in Supplementary material.

Figure 5. Anatase spectra on samples E1A and M4A: a) Raman with active modes, b) GI XRD with assignation and crystal planes.

Figure 6. XRD diffractograms of the titanium layers deposited by electron beam evaporation and magnetron sputtering, assignation and crystal planes presented below,

Figure 7. Structural parameters of the; a) relative ratio of the hcp Ti reflections [002] and [103] (dimensionality insight) and b) Schererr crystallites size.

Figure 8. Scheme showing different titanium thin film morphology exhibit different susceptibility to anodization, i.e. the existence of preferred routes and spots for electrochemical etching, and relation to derived titania morphology as a consequence of the starting morphology. From left to right: i) worstcase scenario occur in the case of microstructure consisting of 1D Ti particles which yields porous nanostructure due to multiple electrolyte penetration points in the course of the anodization; ii) 2Dtype of Ti particles and iii) generally non-isotropic Ti particles yield various blends of porous, wormhole and NT products, also considered as porous; iv) bigger isotropic Ti particles yield favourable NT on micro level with unfavourable cracks on macrolevel, while finally v) smaller isotropic Ti particles yield favourable micro and macro homogeneous titania NT layer. 
Figure 9. Micrographs of the titanium layers deposited by electron beam evaporation; a) surface E1, b) surface E2 and c) surface E3, and by magnetron sputtering; d) surface M4, d) surface M5 and d) surface M6.

Figure 10. a) Anodization current vs time plot; scale inserted; b) micrograph of the excess etching: NT etched trough deposited titanium to the TCO ZnO:Al, c) UV-VIS transmittance of anodized and thermally treated samples; scale inserted.

Figure 11. XRD diffractograms of the titanium thin films samples MAZO, MFTO and MITO deposited by magnetron sputtering on top of AZO, FTO and ITO; assignation and crystal planes presented below, Inset: Ti Schererr crystallites size.

Figure 12. Micrographs of the titanium thin films deposited by magnetron sputtering on top of a) AZO - surface MAZO, b) FTO - surface MFTO and c) ITO - surface MITO, and nanostructures anodized thereof d) AZO - sample MAZOA, e) FTO - sample MFTOA and f) ITO - sample MITOA.

\section{Tables}

Table 1. Titanium deposition conditions; electron beam evaporation conditions, magnetron sputtering conditions.

Table 2. Anodization process parameters and the outcome of the nanostructured $\mathrm{TiO}_{2}$ layer. 
Table 1. Titanium deposition conditions; electron beam evaporation conditions, magnetron sputtering conditions.

\begin{tabular}{cccccccc}
\hline \multirow{2}{*}{$\begin{array}{c}\text { Substrate } \\
\text { No }\end{array}$} & \multicolumn{3}{c}{ Electron beam evaporation } & \multicolumn{5}{c}{ Magnetron sputtering } \\
\cline { 2 - 8 } & $\begin{array}{c}\text { Base } \\
\text { pressure } \\
/ \mathrm{Pa} \cdot 10^{-4}\end{array}$ & $\begin{array}{c}\text { Depos. } \\
\text { rate } \\
/ \AA \mathrm{s}^{-1}\end{array}$ & $\begin{array}{c}\text { Substrate } \\
\text { preheat } \\
/{ }^{\circ} \mathrm{C}\end{array}$ & $\begin{array}{c}\text { Cell } \\
\text { pressure } \\
/ \mathrm{Pa} \cdot 10^{-5}\end{array}$ & $\begin{array}{c}\text { Argon } \\
\text { pressure } \\
/ \mathrm{Pa}\end{array}$ & $\begin{array}{c}\text { Depos. } \\
\text { power } \\
/ \mathrm{W}\end{array}$ & $\begin{array}{c}\text { Substrate } \\
\text { temp. } \\
/{ }^{\circ} \mathrm{C}\end{array}$ \\
\hline E1 & 6.6 & 8.0 & 230 & - & - & - & - \\
E2 & 6.6 & 1.0 & 230 & - & - & - & - \\
E3 & 6.6 & 7.5 & RT & - & - & - & - \\
\hline M4 & - & - & - & 1 & 0.5 & 60 & RT \\
M5 & - & - & - & 1 & 0.5 & 60 & RT \\
M6 & - & - & - & 1 & 0.5 & 60 & RT \\
\hline
\end{tabular}

$\mathrm{E}$ - electron beam evaporation, $\mathrm{M}$ - magnetron sputtering

Table 2. Anodization process parameters and outcome of the nanostructured $\mathrm{TiO}_{2}$ layer.

\begin{tabular}{cccc}
\hline \multirow{2}{*}{$\begin{array}{c}\text { Substrate } \\
\text { No }\end{array}$} & \multicolumn{2}{c}{ Anodization parameters } & Nanostructured $\mathrm{TiO}_{2}$ \\
\cline { 2 - 4 } & Etching voltage & $\begin{array}{c}\text { Etching time } \\
/ \text { min }\end{array}$ & NT formation \\
\hline E1A & 30 & 15 & + \\
E2A & 30 & 30 & 0 \\
E3A & 30 & 45 & - \\
\hline M4A & 30 & 60 & + \\
M5A & 30 & 70 & - \\
M6A & 30 & 60 & - \\
\hline
\end{tabular}

E - electron beam evaporation, $\mathrm{M}$ - magnetron sputtering, $\mathrm{A}$ - anodized to $\mathrm{TiO}_{2}$

$(+)$ : favourable NT formation; (-): unfavourable NT; $(0)$ : other nanostructures formed 


\section{Supplementary material}

\section{Figures}

Figure A. Micrographs after FIB cutting; different tilts and magnification of $\mathrm{TiO}_{2} \mathrm{NT}$ anodized on $\mathrm{Ti}$ deposited by magnetron sputtering on top of a) whole area FIB treated on AZO, b) FIB one surface on FTO and c) zoom-in FIB cross section on ITO.

Figure B. Micrographs of tilted layers of titania NT anodized on anodized on Ti deposited by magnetron sputtering derived by: a) cracking of the substrates and the thin films, b) focusing on defects and c) another example of unfavourable porous titania andodization product. 